\title{
Crystal Structure of 2-(2-Pyridyl)-1,3-bis(2-pyridylmethyl)tetrahydropyrimidine
}

\author{
Takashi Yoкоуama, Nobuyuki EтoH and Michio ZeNKI \\ Department of Chemistry, Faculty of Science, Okayama University of Science, \\ Ridai-cho, Okayama 700, Japan
}

The ruthenium(VI) complex with $N, N^{\prime}$-dimethyl$N, N^{\prime}$-bis(2-pyridylmethyl)propylenediamine has been investigated electrochemically. ${ }^{1}$ The analogous ligand $N, N^{\prime}$-bis(2-pyridylmethyl)propylenediamine, which was prepared according to a literature method ${ }^{1}$, formed complexes with various transition metal ions. In the course of the complex formation researches, a waterinsoluble and non-complex forming white product was obtained. Since this product gave colorless prismatic single crystals in an $N, N^{\prime}$-bis (2-pyridylmethyl)propylenediamine solution, the crystal structure was determined.

The X-ray reflections were collected using a Rigaku AFC7R diffractometer. The single-crystal structure solution was carried out using the program SHELXS86. All non-hydrogen atoms were anisotropically refined, and all hydrogen atoms were isotropically refined. Table 1 summarizes the crystal data. The chemical structure of the title compound is illustrated in Fig. 1. Figures 2 and 3 show the labeling and the packing diagrams of the compound. The atomic coordinates and thermal parameters are presented in Table 2. The bond lengths and angles are presented in Table 3.

The product was a diazacrown ether formed by a

Table 1 Crystal and experimental data

Formula: $\mathrm{C}_{21} \mathrm{H}_{23} \mathrm{~N}_{5}$

Formula weight $=345.45$

Crystal size $=0.60 \times 0.20 \times 0.20 \mathrm{~mm}$

Crystal color: colorless

Crystal system: triclinic

Space group: $P \overline{1}$

$Z=2$

$a=10.738(1) \AA$

$\alpha=98.614(10)^{\circ}$

$b=14.697(1)$

$\beta=103.69(1)$

$c=6.233(1)$

$\gamma=89.813(9)$

$V=944.5(2) \AA^{3}$

$D_{\mathrm{x}}=1.215 \mathrm{~g} / \mathrm{cm}^{3}$

Radiation: graphite monochromated $\mathrm{Cu} \mathrm{K}_{\alpha}$ $\mu$ for $\mathrm{Cu} \mathrm{K}_{\alpha}$ radiation $=5.86 \mathrm{~cm}^{-1}$

$2 \theta$ range: $0^{\circ}$ through $120.1^{\circ}$

$R=0.039$

$$
R w=0.053
$$

No. of reflections measured $=2969$

No. of reflections used $=2409$

Measurement: Rigaku AFC7R

Program system: TEXSAN

Structure determination: direct method

Refinement: full matrix least-squares

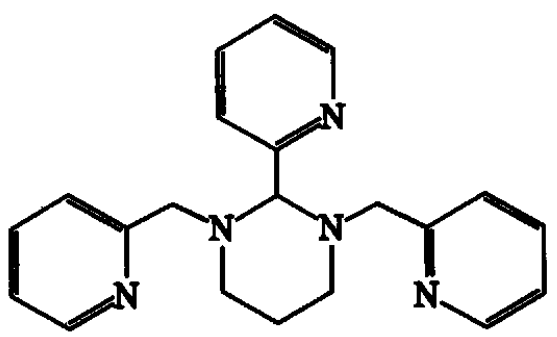

Fig. 1 Chemical structure.

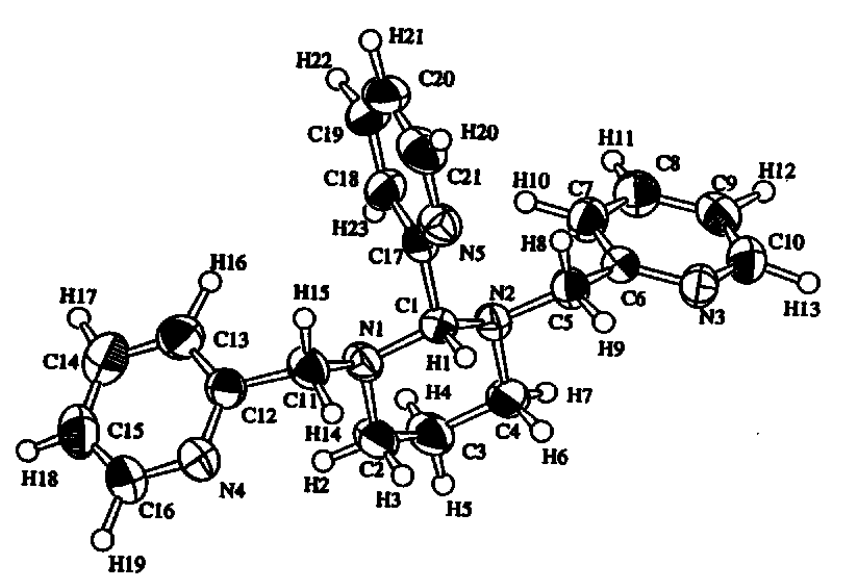

Fig. 2 ORTEP drawing with the atom numbering.

reaction in which a couple of secondary amines in $N, N^{\prime}-$ bis(2-pyridylmethyl)propylenediamine reacted with the aldehyde group of pyridine-2-aldehyde. This ether was used as the starting material for $N, N^{\prime}$-bis(2-pyridylmethyl)propylenediamine. The compound 2-(2-pyridyl)-1,3-bis(2-pyridylmethyl)tetrahydropyrimidine has not been reported. The nitrogen atoms of pyridyl and amino groups could not coordinate to metal ions owing to the formation of tetrahydropyrimidine.

We wish to thank Dr. Genta Sakane, Okayama University of Science, for his helpful advice. We also thank Mr. Takaomi Akamatsu and Mr. Kenji Kohara, Okayama University of Science, for their assistance with the X-ray measurements. 


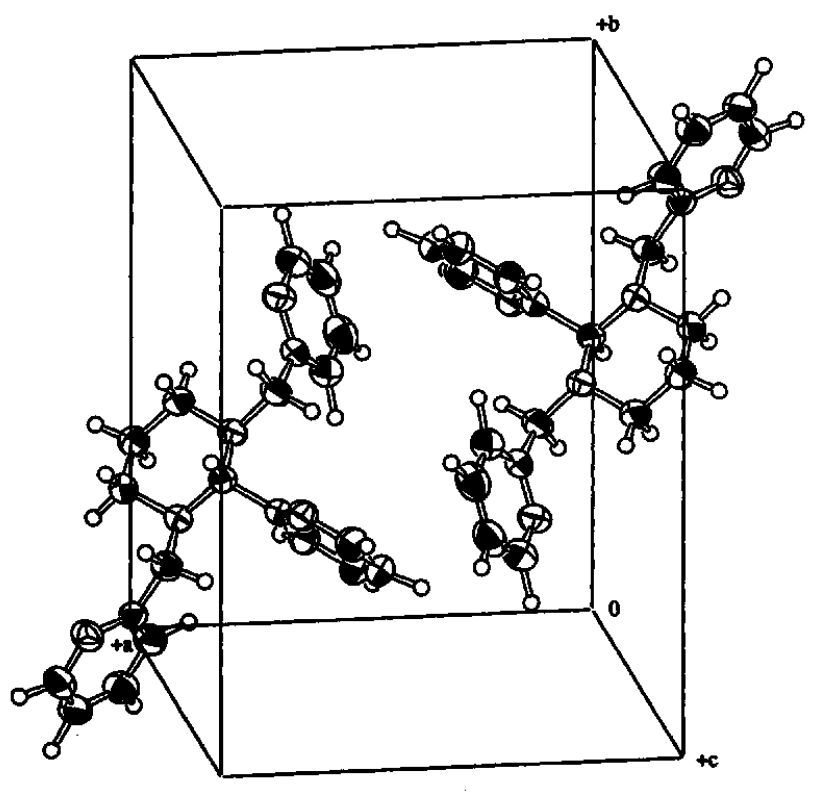

Fig. 3 View of the molecular packings.

Table 2 Atomic coordinates and thermal parameters

\begin{tabular}{lcccc}
\hline Atom & $x$ & $y$ & $z$ & $B_{\text {eq }} / \AA^{2}$ \\
\hline N1 & $0.8109(1)$ & $0.37748(8)$ & $0.1561(2)$ & $3.74(3)$ \\
N2 & $0.9405(1)$ & $0.24564(7)$ & $0.2216(2)$ & $3.38(3)$ \\
N3 & $1.1369(1)$ & $0.05149(8)$ & $0.2322(2)$ & $4.24(3)$ \\
N4 & $0.6825(1)$ & $0.57497(9)$ & $0.0082(2)$ & $4.87(3)$ \\
N5 & $0.6264(1)$ & $0.17901(8)$ & $-0.0179(2)$ & $4.15(3)$ \\
C1 & $0.8198(1)$ & $0.27766(9)$ & $0.0944(2)$ & $3.28(3)$ \\
C2 & $0.9213(2)$ & $0.4279(1)$ & $0.1253(3)$ & $4.65(4)$ \\
C3 & $1.0439(2)$ & $0.3962(1)$ & $0.2606(4)$ & $4.91(4)$ \\
C4 & $1.0524(2)$ & $0.2947(1)$ & $0.1926(3)$ & $4.37(4)$ \\
C5 & $0.9528(2)$ & $0.14685(10)$ & $0.1545(2)$ & $3.73(3)$ \\
C6 & $1.0564(1)$ & $0.10685(9)$ & $0.3184(2)$ & $3.37(3)$ \\
C7 & $1.0635(2)$ & $0.1235(1)$ & $0.5449(3)$ & $4.27(4)$ \\
C8 & $1.1533(2)$ & $0.0802(1)$ & $0.6876(3)$ & $4.73(4)$ \\
C9 & $1.2344(2)$ & $0.0213(1)$ & $0.6000(3)$ & $4.43(4)$ \\
C10 & $1.2235(2)$ & $0.0096(1)$ & $0.3742(3)$ & $4.60(4)$ \\
C11 & $0.6923(2)$ & $0.4116(1)$ & $0.0263(3)$ & $4.27(4)$ \\
C12 & $0.6708(1)$ & $0.5081(1)$ & $0.1267(3)$ & $3.95(3)$ \\
C13 & $0.6432(2)$ & $0.5267(1)$ & $0.3336(3)$ & $5.00(4)$ \\
C14 & $0.6300(2)$ & $0.6164(1)$ & $0.4249(3)$ & $5.75(5)$ \\
C15 & $0.6441(2)$ & $0.6853(1)$ & $0.3065(4)$ & $5.55(5)$ \\
C16 & $0.6695(2)$ & $0.6615(1)$ & $0.1094(4)$ & $5.56(5)$ \\
C17 & $0.7090(1)$ & $0.23000(9)$ & $0.1495(2)$ & $3.26(3)$ \\
C18 & $0.6964(2)$ & $0.2414(1)$ & $0.3680(3)$ & $4.24(4)$ \\
C19 & $0.5954(2)$ & $0.1993(1)$ & $0.4164(3)$ & $5.32(5)$ \\
C 20 & $0.5082(2)$ & $0.1482(1)$ & $0.2458(4)$ & $5.78(5)$ \\
C 21 & $0.5273(2)$ & $0.1391(1)$ & $0.0338(4)$ & $5.37(4)$ \\
\hline
\end{tabular}

$B_{\mathrm{eq}}=(4 / 3) \Sigma_{i} \Sigma_{j} \beta_{i j}\left(a_{i} \cdot a_{j}\right)$.
Table 3 Bond lengths $(\AA)$ and bond angles $\left({ }^{\circ}\right)$

\begin{tabular}{cccc}
\hline $\mathrm{N} 1-\mathrm{C} 1$ & $1.469(2)$ & $\mathrm{N} 1-\mathrm{C} 2$ & $1.465(2)$ \\
$\mathrm{N} 1-\mathrm{C} 11$ & $1.469(2)$ & $\mathrm{N} 2-\mathrm{C} 1$ & $1.467(2)$ \\
$\mathrm{N} 2-\mathrm{C} 4$ & $1.464(2)$ & $\mathrm{N} 2-\mathrm{C} 5$ & $1.464(2)$ \\
$\mathrm{N} 3-\mathrm{C} 6$ & $1.335(2)$ & $\mathrm{N} 3-\mathrm{C} 10$ & $1.340(2)$ \\
$\mathrm{N} 4-\mathrm{C} 12$ & $1.338(2)$ & $\mathrm{N} 4-\mathrm{C} 16$ & $1.340(2)$ \\
$\mathrm{N} 5-\mathrm{C} 17$ & $1.330(2)$ & $\mathrm{N} 5-\mathrm{C} 21$ & $1.343(2)$ \\
$\mathrm{C} 1-\mathrm{C} 17$ & $1.515(2)$ & $\mathrm{C} 2-\mathrm{C} 3$ & $1.500(2)$ \\
$\mathrm{C} 3-\mathrm{C} 4$ & $1.497(2)$ & $\mathrm{C} 5-\mathrm{C} 6$ & $1.505(2)$ \\
$\mathrm{C} 6-\mathrm{C} 7$ & $1.381(2)$ & $\mathrm{C} 7-\mathrm{C} 8$ & $1.373(2)$ \\
$\mathrm{C} 8-\mathrm{C} 9$ & $1.372(2)$ & $\mathrm{C} 9-\mathrm{C} 10$ & $1.369(3)$ \\
$\mathrm{C} 13-\mathrm{C} 12$ & $1.502(2)$ & $\mathrm{C} 12-\mathrm{C} 13$ & $1.379(2)$ \\
$\mathrm{C} 15-\mathrm{C} 16$ & $1.377(3)$ & $\mathrm{C} 14-\mathrm{C} 15$ & $1.367(3)$ \\
$\mathrm{C} 18-\mathrm{C} 19$ & $1.366(2)$ & $\mathrm{C} 17-\mathrm{C} 18$ & $1.386(2)$ \\
$\mathrm{C} 20-\mathrm{C} 21$ & $1.372(3)$ & & $1.366(3)$ \\
& & & \\
$\mathrm{C} 1-\mathrm{N} 1-\mathrm{C} 2$ & $111.6(1)$ & $\mathrm{C} 1-\mathrm{N} 1-\mathrm{C} 11$ & $111.4(1)$ \\
$\mathrm{C} 2-\mathrm{N} 1-\mathrm{C} 11$ & $109.3(1)$ & $\mathrm{C} 1-\mathrm{N} 2-\mathrm{C} 4$ & $112.1(1)$ \\
$\mathrm{C} 1-\mathrm{N} 2-\mathrm{C} 5$ & $110.8(1)$ & $\mathrm{C} 4-\mathrm{N} 2-\mathrm{C} 5$ & $108.7(1)$ \\
$\mathrm{C} 6-\mathrm{N} 3-\mathrm{C} 10$ & $117.3(1)$ & $\mathrm{C} 12-\mathrm{N} 4-\mathrm{C} 16$ & $117.0(1)$ \\
$\mathrm{C} 17-\mathrm{N} 5-\mathrm{C} 21$ & $116.6(1)$ & $\mathrm{N} 1-\mathrm{C} 1-\mathrm{N} 2$ & $110.4(1)$ \\
$\mathrm{N} 1-\mathrm{C} 1-\mathrm{C} 17$ & $108.5(1)$ & $\mathrm{N} 2-\mathrm{C} 1-\mathrm{C} 17$ & $108.8(1)$ \\
$\mathrm{N} 1-\mathrm{C} 2-\mathrm{C} 3$ & $110.5(1)$ & $\mathrm{C} 2-\mathrm{C} 3-\mathrm{C} 4$ & $108.8(1)$ \\
$\mathrm{N} 2-\mathrm{C} 4-\mathrm{C} 3$ & $110.4(1)$ & $\mathrm{N} 2-\mathrm{C} 5-\mathrm{C} 6$ & $112.2(1)$ \\
$\mathrm{N} 3-\mathrm{C} 6-\mathrm{C} 5$ & $116.5(1)$ & $\mathrm{N} 3-\mathrm{C} 6-\mathrm{C} 7$ & $122.0(1)$ \\
$\mathrm{C} 5-\mathrm{C} 6-\mathrm{C} 7$ & $121.4(1)$ & $\mathrm{C} 6-\mathrm{C} 7-\mathrm{C} 8$ & $119.7(1)$ \\
$\mathrm{C} 7-\mathrm{C} 8-\mathrm{C} 9$ & $118.7(2)$ & $\mathrm{C} 8-\mathrm{C} 9-\mathrm{C} 10$ & $118.4(2)$ \\
$\mathrm{N} 3-\mathrm{C} 10-\mathrm{C} 9$ & $123.8(1)$ & $\mathrm{N} 1-\mathrm{C} 11-\mathrm{C} 12$ & $1102(1)$ \\
$\mathrm{N} 4-\mathrm{C} 12-\mathrm{C} 11$ & $116.9(1)$ & $\mathrm{N} 4-\mathrm{C} 12-\mathrm{C} 13$ & $121.8(1)$ \\
$\mathrm{C} 11-\mathrm{C} 12-\mathrm{C} 13$ & $121.2(1)$ & $\mathrm{C} 12-\mathrm{C} 13-\mathrm{C} 14$ & $119.7(2)$ \\
$\mathrm{C} 13-\mathrm{C} 14-\mathrm{C} 15$ & $118.8(2)$ & $\mathrm{C} 14-\mathrm{C} 15-\mathrm{C} 16$ & $118.2(2)$ \\
$\mathrm{N} 4-\mathrm{C} 16-\mathrm{C} 15$ & $124.5(2)$ & $\mathrm{N} 5-\mathrm{C} 17-\mathrm{C} 1$ & $117.6(1)$ \\
$\mathrm{N} 5-\mathrm{C} 17-\mathrm{C} 18$ & $122.7(1)$ & $\mathrm{C} 1-\mathrm{C} 17-\mathrm{C} 18$ & $119.7(1)$ \\
$\mathrm{C} 17-\mathrm{C} 18-\mathrm{C} 19$ & $119.5(2)$ & $\mathrm{C} 18-\mathrm{C} 19-\mathrm{C} 20$ & $118.6(2)$ \\
$\mathrm{C} 19-\mathrm{C} 20-\mathrm{C} 21$ & $118.2(2)$ & $\mathrm{N} 5-\mathrm{C} 21-\mathrm{C} 20$ & $123.8(2)$ \\
\hline & & & \\
\hline
\end{tabular}

\section{References}

1. C.-M. Che, W.-T. Tang and C.-K. Li, J. Chem. Soc., Dalton Trans., 1990, 3735.

(Received June 12, 1995)

(Accepted August 3, 1995) 